\section{Differential inhibition and stimulus generalization cannot account for value transfer in simultaneous discrimination learning by pigeons: Reply to Aitken}

\author{
THOMAS R. ZENTALL, BRIGETTE R. DORRANCE, \\ and TRICIA S. CLEMENT \\ University of Kentucky, Lexington, Kentucky
}

\begin{abstract}
Aitken (1999) argues that, in a simultaneous discrimination, reports of the transfer of value from the positive to the negative stimulus can be more readily explained in terms of an artifact produced by the procedure in which differential inhibition accrues to the negative test stimuli during training, together with stimulus generalization (similarity between the positive and negative stimuli). We argue that (1) there is little evidence for differential inhibition, and it often occurs in the wrong direction; (2) value transfer can be demonstrated when differential value is established to the positive stimuli after discrimination training, when differential inhibition is not likely to be a factor; and (3) on both logical and empirical grounds, stimulus similarity does not provide an adequate account of the transfer of value from the positive to the negative stimulus (i.e., the strongest evidence for value transfer occurs when there is least stimulus similarity). We propose that value transfer occurs whenever there is relatively little experience with the negative stimuli. However, when there is extended experience with the negative stimuli, contrast will be found.
\end{abstract}

It has been proposed that, in a simultaneous discrimination, some of the value of the positive stimulus transfers to the negative stimulus (Fersen, Wynne, Delius, \& Staddon, 1991). Since then, considerable support for value transfer in pigeons has been reported (Clement, Weaver, Sherburne, \& Zentall, 1998; Dorrance, Kaiser, \& Zentall, 1998; Dorrance \& Zentall, 1999; Zentall \& Sherburne, 1994; Zentall, Sherburne, Roper, \& Kraemer, 1996).

The typical procedure for demonstrating value transfer involves training with two simultaneous discriminations in which the probability of reinforcement associated with the two positive stimuli is different (e.g., A100B0 and C50D0, where the letters represent arbitrary stimuli counterbalanced to control for stimulus preference and the numbers following each letter represent the probability of reinforcement associated with responses to each of the stimuli). Following training, when the pigeons are given a choice between the two negative stimuli ( $B$ and $D)$,

Preparation of this article was facilitated by Grants MH55118 and MH59194 from the National Institute of Mental Health. Send correspondence to T. R. Zentall, Department of Psychology, University of Kentucky, Lexington, KY 40506-0044 (e-mail: zentall@pop.uky.edu). they typically choose the one $(B)$ that has been paired in training with the higher valued positive stimulus (A).

To argue against the possibility that the preference for $B$ over $D$ might result from the greater number of nonreinforced responses to $\mathrm{D}$ than to $\mathrm{B}$, we have noted two findings: First, perhaps surprisingly, the proportion of total "errors" made to D is generally not significantly different from the proportion of total errors made to B. Acquisition is generally rapid and preexperimental stimulus preferences account for many of the errors made. Second, the correlation between the proportion of errors made to $D$ and the magnitude of the preference from $B$ is typically negative. One might expect it to be positive if differential inhibition to the two negative stimuli were responsible for the preference for B over D that has been found.

Aitken (1999) has argued that the evidence against differential inhibition as an account of the effects found is not convincing. First, he notes that other individual differences unrelated to differential inhibition may account for the within-experiment negative correlation between proportion of responses to $D$ in training and the preference for B in test. Second, he notes that, between experiments, there is actually a positive correlation between proportion of responses to $D$ in training and choice of $B$ in test. Third, he proposes that residual evidence for value transfer may result from stimulus generalization between the positive and negative stimuli that is produced by their physical similarity rather than by their simultaneous presentation. We will address each of these criticisms in turn.

\section{Differential Inhibition in Training}

First, as already noted, the two discriminations were acquired at rates that typically did not differ significantly. In the three articles cited by Aitken (1999; i.e., Dorrance et al., 1998; Zentall \& Sherburne, 1994; Zentall et al., 1996) there was a total of seven experiments involving differential probabilities of reinforcement associated with the positive stimuli, and in six of those seven experiments there was a nonsignificant (often $F<1$ ) difference in the rate of acquisition between the two discriminations. It could be that the training procedure that we used was not sensitive enough to detect a significant difference in errors to the two discriminations; however, it was certainly sensitive enough to detect the differential transfer of value from the positive to the negative stimuli (i.e., the preference for B over D on test trials, which averaged $82.7 \%$ in Dorrance et al., 1998). More directly relevant to the differential-inhibition hypothesis is that in the seven experiments noted, there were 11 subjects that actually made more errors to $B$ than to $D$ during training, and 10 of those 11 subjects showed a preference for $B$ in test. In fact, the mean preference for B was $80.4 \%$ for those 11 subjects. 


\section{Negative Correlation Between Proportion of Responses to $D$ in Training and Choice of $B$ in Test}

Aitken (1999) suggests that it is inappropriate to use the negative correlation between the proportion of error responses to $\mathrm{D}$ and the preference for $\mathrm{B}$ over $\mathrm{D}$ in test as an indication that differential inhibition is not responsible for the test effect. He notes that slow learners might make proportionally more responses to $\mathrm{D}$; yet, being slow to learn, they may also be less affected by each nonreinforced response, and thus they may show a smaller preference for B over D.

The assumption made by Aitken (1999) is that slow learners may not learn as well as fast learners. But the best measure of the degree of learning is discrimination accuracy at the time of test, and all pigeons were trained to the same high criterion. Furthermore, Aitken assumes that the pigeons that acquired the discriminations more slowly also showed a higher proportion of their error responses to D. Actually, there is no reason to believe that the slow learners would, in general, learn one of the discriminations proportionally slower than the other discrimination, as compared with the fast learners. In fact, for the four experiments reported by Dorrance et al. (1998), the mean correlation between rate of acquisition (total errors) and proportion of total errors made to $D$ was essentially zero $(r=-.04)$.

\section{Positive Correlation Between Proportion of Responses to $D$ in Training and Choice of $B$ in Test}

Aitken (1999) notes that if the data from Dorrance et al. (1998) are presented on an experiment-wise basis (four experiments, with the data from the two groups in Experiment 4 presented separately), there is in fact a strong positive correlation between those measures among the experiments (see his Figure 1). The assumption is that differences in the procedures among the four experiments are responsible for differences in the proportion of errors made to $D$ in training. But Aitken is not specific about what might be responsible for these differences in training, and whatever post hoc account one might be able to propose for the differences across experiments in the proportion of errors made to $D$ in training, there was also a rather large difference in that measure for the two counterbalancing groups in Experiment 4, for which the procedures were exactly the same.

Aitken (1999) may be correct that differential inhibition can play a role in the degree to which $B$ is preferred over $\mathrm{D}$ in test; however, Aitken also acknowledges that differential inhibition cannot account for all of the preference for $B$ over $D$ in test. When pigeons show no difference in errors to B versus D in training (Dorrance et al., 1998, Experiment 3), they still show a strong preference (of almost $70 \%$ ) for B over D in test.

\section{Stimulus Generalization}

Aitken (1999) proposes that stimulus generalization can account for the residual transfer effects reported by Dorrance et al. (1998). Of course, one must distinguish here between value transfer, which is a form of spatialtemporal stimulus generalization, and the more typical stimulus generalization proposed by Aitken, in which similarity between stimuli (independently of their spatial and temporal contiguity) might be responsible for the transfer of value between stimuli. Aitken's argument is that although each of the four hues used in those experiments served equally often as the A, B, C, and D stimuli, the pairs of stimuli assigned to each discrimination were not randomly assigned. This nonrandom assignment of stimuli to pairs was designed to maximize the possibility of finding preferences on test trials by reducing the likelihood of stimulus generalization when novel pairings of the stimuli were presented. Thus, test trials always involved hues with a high degree of spectral separation, such as red and green. To ensure such maximal spectral separation of the test stimuli, the training pairs often involved distinctive stimuli that were spectrally closer together than the test pairs. Aitken suggests that those training stimuli were not of sufficiently different spectral value to rule out stimulus generalization between them.

There are a number of arguments against the notion that this kind of stimulus generalization might serve as the basis for the preference for B over D in test. First, stimulus generalization is an empirical phenomenon. It can be defined as the (relative) probability of a response occurring to a stimulus, following training with a different stimulus. Generalization tests have generally been conducted with the presentation of novel stimuli following single stimulus training (see, e.g., Guttman \& Kalish, 1956). For example, when Guttman and Kalish trained pigeons with a green hue (similar to the green hue used by Dorrance et al., 1998) and then tested them with a variety of hues, they found that the probability of a pecking response was very low to blue and yellow hues (similar to those used by Dorrance et al.). Thus, generalization gradients along the spectral dimension appear to be relatively steep for pigeons. Furthermore, when training involves a discrimination between a positive and a negative stimulus, the postdiscrimination gradients tend to be even steeper than following single-stimulus training (Hanson, 1959). In fact, one could argue that the effect of discrimination training is to virtually eliminate generalization between the positive and the negative stimuli. The training criterion used in value transfer experiments should ensure that there is very little generalization between the two discriminative stimuli. But it is also possible that the simultaneous discrimination procedure masks some stimulus generalization.

More direct evidence against the role of stimulus generalization can be found in an experiment in which more complete counterbalancing of stimuli was used (Dorrance et al., 1998, Experiment 4). If generalization from red to yellow and from green to blue were responsible for the preference for $B$ over $D$ found in the value transfer experiments, one would expect the preference for $B$ over D to be smaller (or actually reversed) when such generalization occurs between discriminations (e.g., 
when one discrimination involves red as the positive stimulus and the other discrimination involves yellow as the negative stimulus; see also Zentall \& Sherburne, 1994, for a similar argument). In fact, contrary to prediction, that condition (Experiment 4, Group R/B) produced the largest preference for B over D $(96.9 \%)$ found by Dorrance et al. Thus, neither differential inhibition to the two negative stimuli, nor stimulus generalization of the type suggested by Aitken (1999), provides an adequate account of the value transfer effects reported by Dorrance et al. and others.

\section{Postdiscrimination Value Transfer}

The evidence for value transfer reported by Dorrance et al. (1998) is further supported by other findings of value transfer in which hypothesized differential inhibition cannot play a role. For example, Zentall et al. (1996, Experiment 3) trained pigeons on two discriminations in which the probability of reinforcement associated with the two positive stimuli was the same (A50B0, C50D0). Following acquisition, pigeons were trained on a third discrimination involving the two positive stimuli from original training $(\mathrm{A} 100 \mathrm{C} 0)$. In this case, there should be no differential inhibition because the two discriminations involve identical schedules of reinforcement; yet on test trials, the pigeons still showed a significant preference for B over D. Similarly, Dorrance and Zentall (1999) have shown that nonreinforced responding to presentations of A alone following A50B0, C50D0 training can result in a strong preference for $D$ over $B$.

\section{Negative Value Transfer (or Positive Contrast)}

Aitken (1999) also notes that we have not been able to find evidence for the transfer of negative value from the negative stimulus to the positive stimulus (Clement et al., 1998). In that research, we first asked whether following discrimination training involving A100B0, C100D50 we would find a preference for $C$ over $A$. The reasoning was that as a result of such training, $B$ might draw more value away from $A$ than $D$ would draw from $C$. We found no consistent evidence of preference for $\mathrm{A}$ or $\mathrm{C}$. But we also noted that, whereas in the case of the positive value transfer design, in which the greater value given to A than to $\mathrm{C}$ was experienced on most trials by the pigeons, in the case of the negative value transfer design, very few responses were made to either B or to D; thus, the source of the manipulation (more reinforced responses to D50 than to B0) might not have been sufficiently experienced. To overcome this problem, we gave the pigeons explicit training with the values of $B$ and $D$ by presenting those stimuli by themselves on additional trials. When we then tested with the $\mathrm{A}$ and $\mathrm{C}$ stimuli, we found that the pigeons showed a strong preference for $A$ over $C$. Not only did we fail to find negative value transfer, but we found strong evidence for the opposite, positive contrast. Thus, when we added single-stimulus presentations of $B$ and $\mathrm{D}$ to the A100B0, C100D50 discriminations, the lesser value of $B$ (relative to $D$ ) actually enhanced the value of $A$ (relative to $C$ ). Our conclusion in that article was that in a simultaneous discrimination, the positive stimulus may transfer some of its value to the negative stimulus, but the negative stimulus may also enhance the value of the positive stimulus by way of positive contrast.

\section{Negative Contrast}

An alternative interpretation of the positive contrast effect reported by Clement et al. (1998) is that value transfer will occur when experience with the negative stimuli is limited (e.g., in a typical easy simultaneous discrimination; Zentall \& Sherburne, 1994), whereas contrast will occur when extended experience with the negative stimuli is provided (e.g., when, in addition to discrimination training, there is also single-stimulus experience with the negative stimuli; Clement et al., 1998). To test this hypothesis, we are currently conducting an experiment with the value transfer design (i.e., A 100B0, C50D0) in which we are providing more direct experience with the negative stimuli (i.e., by including single-stimulus B0 and D0 trials). If extensive experience with the negative stimuli is sufficient to produce contrast, we should find that the inclusion of B0 and D0 trials in training will reverse the preference in test (pigeons will prefer $D$ over B). The bottom line is that it may be possible to demonstrate contrast effects following simultaneous discrimination training, but in the typical case in which experience with the negative stimulus is limited, the positive stimulus will transfer some of its value to the negative stimulus.

\section{REFERENCES}

AitKEN, M. R. F. (1999). Alternative accounts are preferable to value transfer theory: Commentary on Dorrance, Kaiser, and Zentall (1998). Animal Learning \& Behavior, 27, 490-493.

Clement, T. S., Weaver, J. E., Sherburne, L. M.. \& Zentall, T. R. (1998). Simultaneous discrimination learning in pigeons: Value of $\mathrm{S}-$ affects the relative value of its associated S+. Quarterly Journal of Experimental Psychology, 51B, 363-378.

Dorrance, B. R., Kaiser, D. H., \& ZenTall. T. R. (1998). Value transfer in a simultaneous discrimination by pigeons: The value of the $\mathrm{S}^{+}$ is not specific to the simultaneous discrimination context. Animal Learning \& Behavior, 26, 257-263.

Dorrance, B. R., \& ZENTALL, T, R. (1999). Within-event learning contributes to value transfer in simultaneous instrumental discriminations by pigeons. Animal Learning \& Behavior, 27, 206-210.

Fersen, L. von, Wynne. C. D. L., Delius, J. D., \& Staddon, J. E. R. (1991). Transitive inference formation in pigeons. Journal of Experimental Psychology: Animal Behavior Processes, 17, 334-341.

GutTman, N., \& Kalish, H. I. (1956). Discriminability and stimulus generalization. Journal of Experimental Psychology. 51, 79-88.

HANSON, H. M. (1959). Effects of discrimination training on stimulus discrimination. Journal of Experimental Psychology, 58, 321-333.

Zentall, T. R., \& Sherburne, L. M. (1994). Transfer of value from S+ to $\mathrm{S}-$ in a simultaneous discrimination. Journal of Experimental Psychology: Animal Behavior Processes, 20, 176-183.

Zentall, T. R., Sherburne, L. M., Roper, K. L., \& Kraemer, P. J. (1996). Value transfer in a simultaneous discrimination appears to result from within-event Pavlovian conditioning. Journal of Experimental Psychology: Animal Behavior Processes, 22, 68-75.

(Manuscript received June 21, 1999; revision accepted for publication August 3,1999 .) 